\title{
The value of PET/CT to assess clinically negative necks
}

\author{
Brendan C. Stack • Val J. Lowe
}

Published online: 11 March 2012

(C) Springer-Verlag 2012

\section{Dear Editor,}

We read Ozer's article [1] from the Ohio State University with great interest. This is a large sample size and the authors are to be congratulated for amassing such a large series. The results of this study should be taken cautiously as they reflect some shortcomings in the study design which were unavoidable. Among them are its retrospective collection of subjects, no detailed correlation between neck nodal levels and imaging or pathology data, a treatment bias as to which N0 necks would be dissected, and no dedicated PET/ CT imaging of exclusively the neck are reported as being used. In spite of these shortcomings, the reported negative predictive value (NPV) is still high in the N0 neck.

This issue with PET scanning for the clinical N0 neck is not with false positives. They will likely be more carefully scrutinized with ultrasound guided biopsy or will proceed to neck dissection. The issue is whether there are false negative scans. Because the power of the PET scan calling a neck negative is the opportunity to safely avoid elective neck dissection or radiation and save money and avoid complications. For this reason we need to know with the best possible science what is the NPV of PET/CT for the N0 neck.

There is no lack of data to collect and meta-analyze to answer this question. There are numerous single institution series available for this purpose. It is only with a prospective study can the biases of elective neck dissection deci-

B. C. Stack $(\bowtie)$

University of Arkansas for Medical Sciences,

Little Rock, USA

e-mail: BStack@uams.edu

V. J. Lowe

Mayo Clinic, Rochester, MN, USA sion making be eliminated. We recognized this problem in our paper in 2001 and spent years engaging the US National Institutes of Health (NIH) through the R01 mechanism before we were fortunate to receive support through the American College of Radiology Imaging Network (ACRIN). ACRIN is an NIH sponsored cooperative group which focuses upon imaging in the evaluation and treatment of cancer patients. Recently, the ACRIN has been merged with the Eastern Cooperative Oncology Group (ECOG) and will have an exciting future combining advanced imaging with the latest advancements in medical oncology.

ACRIN 6685, "A multicenter trial of FDG PET/CT staging of head and neck cancer and its impact on the NO neck surgical treatment in head and neck cancer patients," was designed to definitively answer the issues raised by Ozer et al. and many other groups. The main objective of our study is to determine the NPV for the N0 neck. Participants will include 292 prospectively enrolled new head and neck cancer patients being considered for resection with at least one side of the neck being planned for dissection clinically N0 with at least a $30 \%$ chance of occult nodal metastasis. More details can be found at http://www.acrin.org/6685_ protocol.aspx

We applaud the journal publishing scientific works like Ozer et al. and hope that through ACRIN 6685 we may collect the data necessary to answer the question about PET/ CT utility in the management of the NO neck.

\section{Reference}

1. Ozer E, Naiboglu B, Meacham R, Ryoo C, Agrawal A, Schuller DE (2012) The value of PET/CT to assess clinically negative necks. Eur Arch Otorhinolaryngol [Epub ahead of print] 